\title{
Eye movements measured with a solid state photodiode matrix
}

\author{
H. P. WILKINSON \\ University of Nottingham, University Park, Nottingham NG7 2RD, England
}

\begin{abstract}
The apparatus described uses the corneal reflex technique to measure horizontal and vertical eye movements with a metal oxide semiconductor matrix array of 64 by 64 photodiodes. A simple optical system forms a reflex image on the array and also determines the device range, which can vary from $\pm 25 \mathrm{deg}$ to $\pm 3 \mathrm{deg}$; sensitivity is 50 arc min and 6 arc min, respectively. Mounted on a simple lightweight headband complete with universal mechanical adjusters, the instrument weighs less than $100 \mathrm{~g}$, excluding connecting cable, and samples eye movements every $5 \mathrm{msec}$.
\end{abstract}

Of the many techniques available for monitoring eye movements, measurement of the position of a spot of light reflected from the front surface of the eye may be combined with recent advances in optical electronics to produce a compact eye movement monitor. The device described below uses this corneal reflex technique in conjunction with a sophisticated piece of electronic hardware consisting of an array of light-sensitive diodes and associated driving waveform generator and decoding circuitry.

The mechanical mounting assembly is constructed from readily available materials, and the complete monitor can be built using normal workshop facilities and expertise.

This instrument has its origins in the technique first reported by Mackworth and Mackworth (1958), in which a head-mounted camera records the corneal reflex and presents it as a light spot representing point of regard on a scene viewed by the subject. Although at present this device only records reflex position and not the viewed scene, it does so by means of a miniature solid state video camera specially developed for this purpose. The heart of this camera is a two-dimensional array of 64 by 64 photodiodes manufactured by Integrated Photomatrix Ltd., Dorchester, Dorset, United Kingdom.

Device sensitivity is varied by appropriate optical adjustment; the mechanical mounting, with its universal adjustments, facilitates setting up and use with a variety of subjects. The unit is lightweight for subject comfort and device accuracy.

\section{OPTICAL DESIGN}

By illuminating the eye with parallel light, movement $h_{\theta}$ of the corneal reflex due solely to eye rotation is given by the formula (after Ditchburn \& Ginsborg, 1953):

$$
\mathrm{h}_{\theta}=\left(\mathrm{r}_{\mathrm{c}}-\mathrm{r}_{\mathrm{e}}\right) \sin \theta
$$

where $r_{c}=$ radius of curvature of the anterior surface of the cornea, approximately $8 \mathrm{~mm} ; \mathrm{r}_{\mathrm{e}}=$ distance between the apex of the cornea and the instantaneous center of rotation of the eye, approximately $13 \mathrm{~mm}$; $\theta=$ angle of rotation of the eye.

This movement is shown in Figure 1 for a large value of $\theta$, so that an estimate can be made of the maximum range of $\theta$ available. Lens $B$ forms a real image of the reflex on the diode array. This reflex, which is really the virtual image of a distant illuminating source, appears originally to be located at $\mathrm{F}$, the focal point of the cornea, and moves to $I_{\theta}$ as the eye rotates through angle $\theta$. The maximum value of $\theta$ is given approximately by:

$$
\theta_{\max }=\arcsin \left(\mathrm{w} / 2 \mathrm{r}_{\mathrm{e}}\right),
$$

where $\mathrm{w}=\mathrm{LL}$, the corneal width, approximately $11 \mathrm{~mm}$.

Hence $\theta_{\max }=25 \mathrm{deg}$, given that lens $\mathrm{C}$ must interfere minimally with binocular vision and that further limitations are imposed by the corneal geometry in the limbus region, causing distortion of the reflex image. Reflex movement is only linearly related to eye rotation for values of $\theta$ in the range \pm 15 deg because of distortions produced by the shape of the cornea and the sine term in Equation 2. This may be overcome by using a

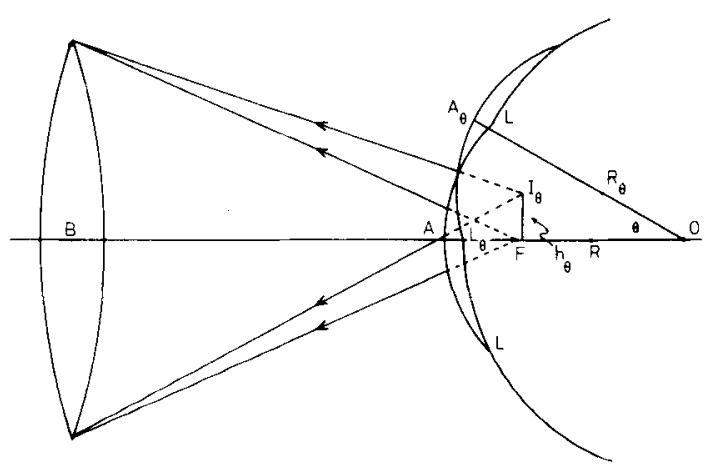

Figure 1. Reflex formation and movement. 
calibration procedure that also allows for individual variations in corneal geometry. A suitable technique that can be adapted for use in two dimensions is described by O'Regan (1978).

\section{CHOICE OF LENS}

The active area of the diode array is $4.8 \mathrm{~mm}^{2}$, so the focal length $f$ of lens $B$ is related to the distance $u$ between corneal apex and lens (AB in Figure 1) by:

$$
u=\left[1+\left(r_{e}-r_{c}\right) / 2.4\right] f-r_{c} / 2 .
$$

In determining the overall device dimensions, an important parameter is the distance $d$ between the diode array and corneal apex, which must be kept small. Using an aspheric acrylic lens of focal length $12 \mathrm{~mm}$, diam $=14 \mathrm{~mm}$, d is less than $50 \mathrm{~mm}$, assuming $\theta_{\max }= \pm 25 \mathrm{deg}$. To achieve a small range of $\theta_{\max }= \pm 3 \mathrm{deg}$, with maximum sensitivity, a $14-\mathrm{mm}$ focal length lens is required. This increases $d$ to $155 \mathrm{~mm}$, which requires the optical path between lens and array to be folded using a small plane mirror so that a completed system remains physically quite small. A shorter focal length lens reduces $u$ to such a small value that eyelash interference with lens B may occur.

The diode array is mounted in a conventional 24-pin dual in-line ceramic package with a small raised square window immediately above the photosensitive area. Clipped over this window is a thin square of aluminum carrying a threaded tube $16 \mathrm{~mm}$ long. Fitting over this tube is another tube bearing a matching interval thread and carrying the lens at one end. This arrangement provides a simple, compact optical system that allows changes in the lens-array separation, thus providing some adjustment of the device range.

A reflex image is produced by illuminating the eye with a small infrared diode Type TIL 35 manufactured by Texas Instruments. The spectral output of this diode matches the region of maximum sensitivity of the MOS array.

\section{TWO-DIMENSIONAL DIODE ARRAY}

The two-dimensional array is a MOS device consisting of a matrix of 64 by 64 photosensitive diodes fabricated on a $6.2-\mathrm{mm}^{2}$ substrate. On-chip circuitry also includes two shift registers that enable access to each of the 4,096 diodes in turn; the total photosensitive area is $4.8 \mathrm{~mm}^{2}$.

Essentially, each diode has a reverse bias voltage applied to it, which causes it to behave like a small capacitor. Any light falling on the diode generates an electric charge that, together with leakage currents, gradually discharge the capacitor; the rate of discharge is proportional to light intensity. If, after a short time, the biasing voltage is reconnected to the diode, a small recharging current flows. This reestablishes the initial reverse bias voltage across the diode. The charging spikes are of the order of picacoulombs in magnitude and a few tens of nanoseconds in duration. By recharging each diode capacitor in turn and amplifying the resultant charge spikes, a voltage waveform is produced whose amplitude is determined by the amount of light falling on each diode in one complete scan, or frame, of the array.

Integrated Photomatrix Ltd. manufactures a complete camera based on the array. Unfortunately, this system does not lend itself to use as a lightweight, head-mounted eye movement monitor. It was necessary to develop a simplified scanning technique and charge-current-tovoltage converter circuit suitable for use as a video preamplifier of the array output, thereby allowing remote connection of the array, along approximately $1.5 \mathrm{~m}$ of cable to the main processing electronics.

\section{ARRAY SCANNING SYSTEM AND VIDEO PREAMPLIFIER}

The array is designed for scanning by one of two techniques involving a minimum of eight separate waveforms, as indicated in Figure 2. On-chip shift registers associated with $\mathrm{X}$ and $\mathrm{Y}$ scanning of the array require -24-V START SCAN pulses together with their own separate $-24-V$ biphase clock waveforms $X_{1}, X_{2}$, $Y_{1}$, and $Y_{2} . R$ and $F$ waveforms switching transistors $R_{t}$ and $F_{t}$, respectively, complete the requirement. The latter waveforms are not strictly necessary and may be omitted by simply connecting $R$ to $-24 \mathrm{~V}$, switching off all transistors $R_{t}$, and connecting $F$ to $0 \mathrm{~V}$, switching on all transistors $F_{t}$. The net result is that the recharge path for the array diodes, formerly via $R_{t}-X_{t}$, becomes $Y_{t}-F_{t}-X_{t}$.

This important change is necessary to reduce to a minimum the number of wires required to connect the head-mounted array and video preamplifier with the main processing electronics. This cable must be as flexible as possible to prevent movement of the headband when the instrument is in use.

The video preamplifier, also shown in Figure 2, uses just two of the high-gain transistors available in an RCA device (CA3095). Although this is a 16-pin DIL package containing other unused transistors, no suitable high-gain discrete devices could be found. The preamplifier includes a small integration capacitor, $C_{i}$, and represents the smallest number of components required for charge-to-voltage conversion of the array output, at the same time producing a low output impedance suitable for driving $1.5 \mathrm{~m}$ of screened cable.

\section{MECHANICAL ADJUSTMENT AND ASSEMBLY}

Previous experience with eye movement monitors clearly demonstrates the need for universal mechanical adjustments, and, with a head-mounted system, weight must be kept as low as possible. This leads to the choice of stainless steel rectangular waveguide for making the $X, Y$, and $Z$ adjusters, together with the mounting stem supporting the small printed circuit boards holding 


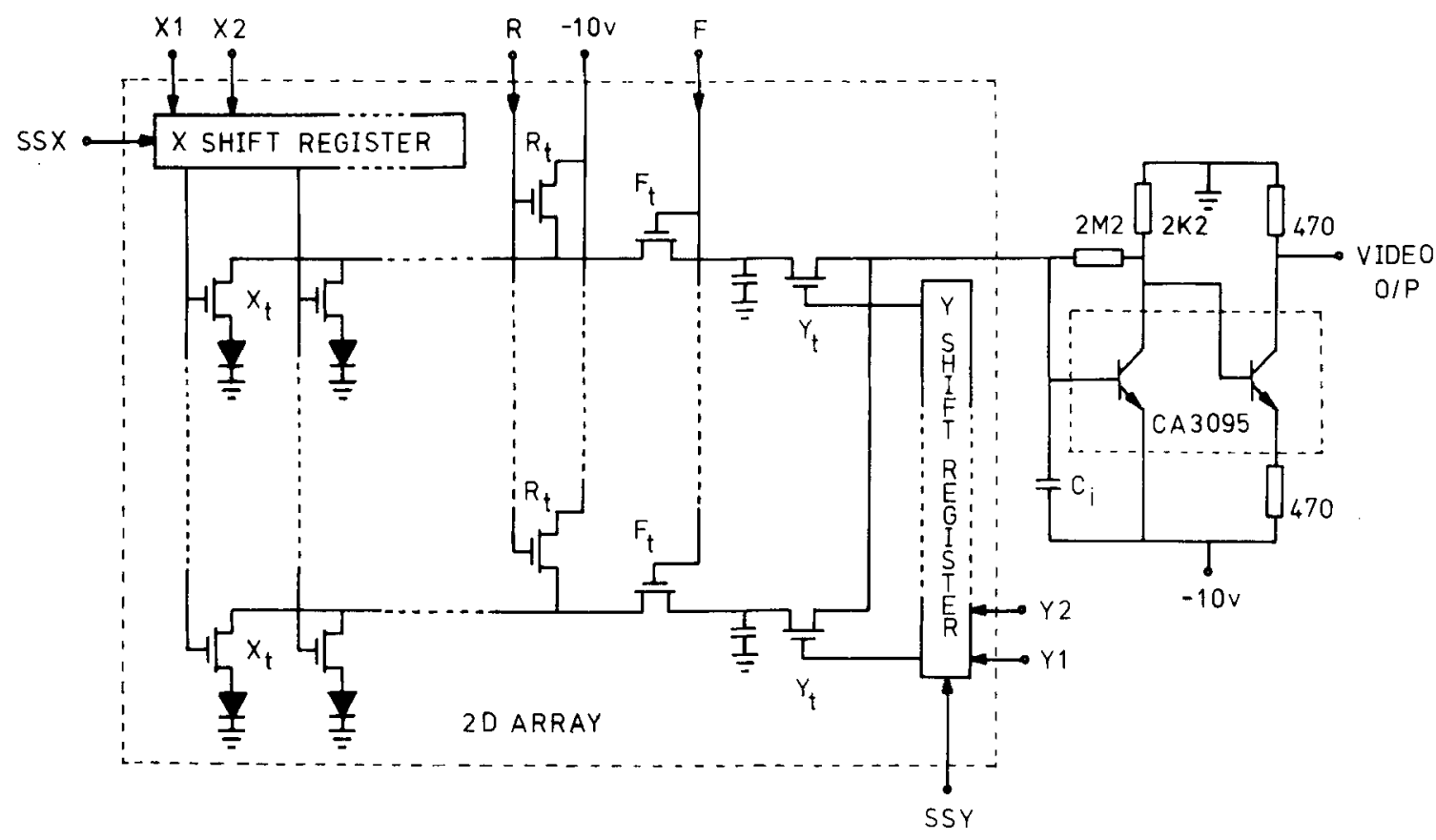

Figure 2. Two-dimensional array and video preamplifier circuit.

preamplifier, diode array, and optics. This material is ideal since it is light yet strong and rigid, may be easily soldered, and acts as electrical screening for wires fed inside the printed circuit board support stem to the DIL connecting socket mounted on the aluminum headband.

One end of the $\mathrm{X}$ adjuster is fitted to a small universal ball joint, allowing the whole assembly to be twisted and turned through a wide range, which facilitates setting up the monitor with a variety of subjects.

The infrared diode is attached to the printed circuit support stem by fine-guage steel wire with electrical connections made to the DIL socket. The completed assembly, including all mechanical and electronic components, weighs less than $100 \mathrm{~g}$ and is shown in Figure 3.

Use of a mouth bite is recommended for precision work because of contaminations of the eye movement output signal due to head movements. A well designed mouth bite, together with a comfortable seating position, reduces head movement contamination to a level well below the device sensitivity (Findlay, 1969).

\section{PROCESSING ELECTRONICS}

A current of $50 \mathrm{~mA}$ through the infrared diode gives sufficient light output to produce a usable video preamplifier output when scanning the array every $5 \mathrm{msec}$. This requires a master clock frequency of $1.3 \mathrm{MHz}$, so CMOS circuitry is used throughout the system except for the cable drivers, which convert the $+12-\mathrm{V}$ logic of the waveform generators to $-24-\mathrm{V}$ logic as required by the array. The final $X$ and $Y$ outputs are obtained by using 741 operational amplifiers, incorporating independent shift and gain controls for each channel. A digital output is also available if required.
A block diagram of the system is shown in Figure 4 which requires little explanation except for the dynamic fault store.

The diode array costs several hundred dollars when bought as a single item and could be considered prohibitively expensive. Some arrays, however, have a small number of faulty diode locations but otherwise function correctly. The substandard arrays are available from the

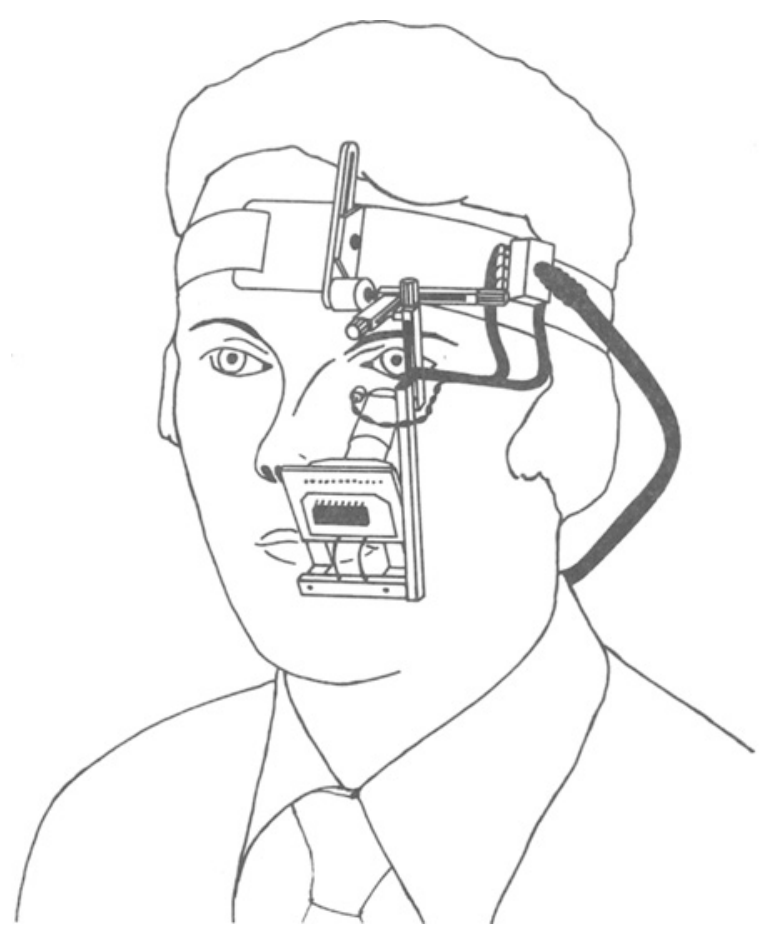

Figure 3. Head assembly showing mechanical construction and mounting. 


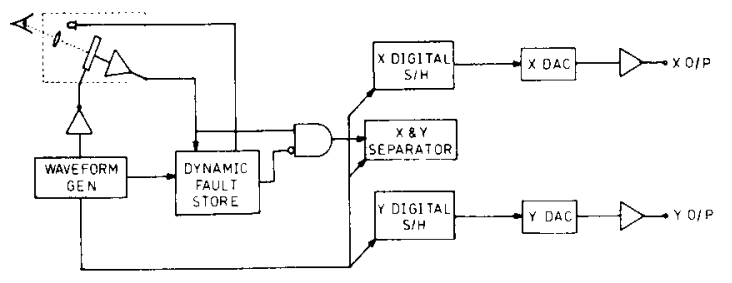

Figure 4. System block schematic.

manufacturer, by negotiation, for approximately $\$ 40$; they are good enough for the present purpose. The number of fault locations varies, but it is typically less than 10 and spreads over no more than 3 or 4 lines. This means that less than $.25 \%$ of the diodes are affected.

If the fault is a disconnection of the array diode, then no video output is available at this point, so it may be ignored. If, on the other hand, the array diode appears to act as a short circuit, then each time an attempt is made to charge the diode at this location, a large voltage pulse appears at the video preamplifier output, even when no light falls on the array.

Faulty outputs of the latter type always occur at the same time during each frame scan. If a 64-bit shift register is associated with the line containing faults, then the faults can be stored as data in the shift register. Thus each of the three or four faulty lines has associated with it a 64-bit shift register that automatically acquires and stores the faults in the first frame scan following power-up. Further data acquisition by the shift registers is then inhibited, and their contents are recirculated each time the appropriate line is interrogated on the array during subsequent scans. Once the faults are stored, current is automatically passed through the infrared diode, producing a light spot on the array representing the subject's point of regard, assuming his head is fixed. The light spot is approximately 10 array diodes in diameter, and suitable integration of the video preamplifier signal allows detection of the spot centroid to within one diode location.

As the integration amplifier output consists of reflex position plus faults and the dynamic fault store provides an output equivalent to faults alone, the appropriate gating of these two signals leaves the reflex position intact prior to separation into its $X$ and $Y$ components.

This extra circuit complexity adds about $\$ 60$ to the total system cost, which is insignificant compared with the price of a perfect array (which can, when available, be substituted directly for the substandard device without further circuit changes). Other faulty arrays may be used, provided they have no greater number of faulty lines than the original and the shift registers are readdressed to the new lines. As faults are stored automatically on power-up, care must be taken not to point the array at windows or room lights, otherwise false information is acquired by the fault store.

\section{DISCUSSION}

Although this eye movement monitor has the inherent shortcomings of any system based on movement of the corneal reflex, its usefulness has been demonstrated in pattern recognition tasks, reading studies, and problem solving. By using a substandard array, the total component cost is kept below $\$ 450$, making it substantially less costly than commercially available products.

Because head-mounted devices must be small and light, the system is best suited to eye movements in a range greater than $\pm 10 \mathrm{deg}$. By careful design using a folded optical path, this range can be readily extended down to $\pm 3 \mathrm{deg}$; a more substantial headband may be required for any developments for covering small eye movements below $\pm 1 \mathrm{deg}$, with a consequent increase in weight.

Device sensitivity, basically $1 / 64$ th of the range, is determined by the diode array pitch. Although other MOS arrays are available (Reticon manufactures a 120 by 120 matrix), substantial improvement in sensitivity can only be made by using charge-coupled (CCD) arrays containing far greater numbers of photosensors on a much finer pitch. Unfortunately, the cost of such CCD arrays, even imperfect ones, is over $\$ 2,000$. CCD devices are available with a 100 by 100 matrix of photosensors incorporated into a solid state camera kit. Suitable modification may produce a system adapted to eye movement measurements, but the coarse matrix pitch still limits sensitivity.

Future development of the present instrument, using a second array, may lead to a miniaturized solid state version of the original eye-mark camera.

Finally, the key features of the instrument described above are (1) inherently wide range both vertically and horizontally, (2) high sample rate, (3) universal mechanical adjustments, (4) lightweight head-mounted assembly, and (5) modest component cost (about \$450).

Young and Sheena (1975) point out the shortcomings of reflex techniques compared with those of other systems. A solid state diode array may not produce an ideal solution to these problems, but its design indicates how developments in the rapidly expanding technology of optoelectronics may lead to smaller, lighter, and cheaper eye movement monitors.

\section{REFERENCES}

Ditchburn, R. W., \& Ginsborg, B. L. Involuntary eye movements during fixation. Journal of Physiology, 1953, 119, 1-17.

Findlay, J. M. The magnitude of translational head movements. Optica Acta, 1969, 16, 65-68.

Mackworth, J. F., \& M Ackworth, N. H. Eye fixations recorded on changing visual scenes by the television eye-maker. Journal of the Optical Society of America, 1958, 48, 439-445.

O'REGAN, K. A new horizontal eye movement calibration method: Subject controlled "smooth pursuit" and "zero drift." Behavior Research Methods \& Instrumentation, 1978, 10, 393-397.

Young, L. R., \& Sheena, D. Survey of eye movement recording methods. Behavior Research Methods \& Instrumentation, 1975, 7, 397-429.

(Received for publication March 27, 1979; revision accepted June $6,1979$. 